\title{
Incidencia de plagas en material poscosecha de nueve cultivares de hortensia tipo exportación, en Antioquia (Colombia)
}

\author{
Incidence of pests in post-harvest material of nine export-type hydrangea \\ cultivars in Antioquia (Colombia) \\ MARÍA DANIELA MOLINA-ACOSTA ${ }^{1}$; SAMIR JULIÁN CALVO²; \\ MAGDA MILENA PALACIO ${ }^{3}$; CARLOS EDUARDO GIRALDO ${ }^{4}$
}

${ }^{1}$ Agrónoma. Universidad Católica de Oriente, Rionegro, Antioquia, Colombia, danielamolina0513@gmail.com, https://orcid.org/0000-00031493-902X. ${ }^{2} \mathrm{Ph}$. D. Grupo de investigación en agronomía y zootecnia - GIAZ, Facultad de Ciencias Agropecuarias, Universidad Católica de Oriente, Sector 3 Cr 46 \#40B-50, Rionegro, Colombia, sjcalvo@uco.edu.co, https://orcid.org/0000-0003-3400-5208. ${ }^{3}$ Ingeniera Agrónoma. M. Sc. Proyecto Ornamentales Antioquia, Instituto Colombiano Agropecuario (ICA), Carrera 45 \#31-03, Bello, Antioquia, Colombia, magdapalaciovilla@gmail.com, https://orcid.org/0000-0001-7646-0108. ${ }^{4}$ M. Sc., Ph. D. Grupo de Investigación de Sanidad Vegetal, Facultad de Ciencias Agropecuarias, Universidad Católica de Oriente, Rionegro, Antioquia, Colombia, cegiral0@gmail.com, https://orcid.org/00000001-6651-3819.

\author{
Autor para correspondencia \\ Carlos Eduardo Giraldo. M. Sc., Ph. D. Gru- \\ po de investigación de sanidad vegetal, \\ Facultad de Ciencias Agropecuarias, Universi- \\ dad Católica de Oriente, Rionegro, Antioquia, \\ Colombia,cegiral0@gmail.com, https://orcid. \\ org/0000-0001-6651-3819.

\section{Citación sugerida} \\ MOLINA-ACOSTA, M. D. CALVO, S. J.; \\ PALACIO, M. M.; GIRALDO, C. E. 2021. In- \\ cidencia de plagas en material poscosecha de \\ nueve cultivares de hortensia tipo exportación, \\ en Antioquia (Colombia). Revista Colombia- \\ na de Entomología 47 (1): e7530. https://doi. \\ org/10.25100/socolen.v47i1.7530 \\ Recibido: 15-feb-2019 \\ Aceptado: 14-dic-2019 \\ Publicado: 23-mar-2021 \\ Revista Colombiana de Entomología \\ ISSN (Print): 0120-0488 \\ ISSN (On Line): 2665-4385 \\ https://revistacolombianaentomologia.univalle.edu.co \\ Open access

\section{(c) (1) (3)(2) BY-NC-SA 4.0}

Publishers: Sociedad Colombiana de Entomología SOCOLEN (Bogotá, D. C., Colombia) https://www.socolen.org.co

Universidad del Valle (Cali, Colombia)

https://www.univalle.edu.co

(C) 2021 Sociedad Colombiana de Entomología SOCOLEN y Universidad del Valle - Univalle
Resumen. El sector floricultor en Colombia genera gran impacto económico y social desde hace más de 40 años. Las hortensias tienen los mayores incrementos en áreas sembradas y exportaciones, pero muy poco se tiene documentado sobre las principales plagas y enfermedades que afectan los cultivos. Este trabajo evaluó las plagas presentes en flores de hortensia inspeccionadas en una comercializadora del oriente antiqueño. Durante cinco meses se evaluaron 6.272 muestras (31.360 flores), de nueve cultivares procedentes de 86 predios en seis municipios. El 8,61 \% de las muestras presentaron algún individuo limitante para su comercialización. Los artrópodos de mayor incidencia $(75,19 \%)$ fueron los ácaros, tanto huevos como formas móviles, y en menor proporción áfidos, trips y babosas. Los cultivares más afectados fueron los de flores azules. El $90 \%$ de los predios $(\mathrm{n}=86)$ presentaron algún evento de interceptación, sin un patrón de afectación espacial asociado al municipio de procedencia. El análisis sugiere que la interceptación por plagas cuarentenarias se asocia al manejo del cultivo y al tratamiento poscosecha, más que a los cultivares o localización geográfica de los mismos. Este trabajo presenta por primera vez información referente a las plagas de mayor incidencia en hortensias tipo exportación, y aporta información que puede ayudar a identificar plagas, sectores y cultivares prioritarios para inspección y evaluación en campo.

Palabras clave: Hydrangea spp., manejo integrado de plagas, floricultura, plagas cuarentenarias, artrópodos, interceptación, Colombia.

Abstract: Floriculture in Colombia has great economic and social impact bringing important profit to the sector for more than 40 years. Recently, the hydrangeas flower market have the largest growths in cultivated areas and exported product, but they also have the highest rates of interceptions at the international ports. Even so, few information about the main pests affecting crops, areas or cultivars has been published. This study evaluated the pests captured on hydrangeas' flowers inspected in a trading company of the Eastern region of Antioquia. A total of 6,272 samples (31,360 flowers), of nine cultivars from 86 farms in six municipalities collected during five months were used. The $8.61 \%$ of the samples had at least one limiting-pest specimen for exportation. The most frequent arthropods were mites, eggs and motile stages, and in less frequency aphids, thrips and slugs. The most affected cultivars were those with blue flowers. The $90 \%$ of the farms $(n=86)$ had at least one event of interception, although there was no spatial pattern associated with municipality. Our data suggest that the interceptions by quarantine pests were associated with the crop and post-harvest management rather than their geographic location or cultivars. This work provides, for the first time, information of the pests with highest incidence in exporting-type Hydrangeas. Although there is no information about the incidence of hydrangeas pests in the crops, these results could help to identify priority areas and cultivars for inspection and evaluation in the field.

Keywords: Hydrangea spp., Integrated Pest Management, floriculture, quarantine pests, arthropods, interception, Colombia.

\section{Introducción}

El sector floricultor ha tenido una gran importancia económica al incrementar las exportaciones y generar divisas desde hace más de 40 años (Arbeláez 1993). Actualmente, Colombia es el primer país proveedor de flores a Estados Unidos y el segundo exportador de ellas a nivel mundial (Valcárcel Calderón 2013). A finales 
de 2016, este gremio tenía un área aproximada de 7.000 ha en producción de flores de exportación, de las cuales según el Instituto Colombiano Agropecuario (ICA), las rosas son las principales especies sembradas con una participación de $37 \%$, seguidas del clavel con un $15 \%$, la hortensia con el $14 \% \mathrm{y}$, finalmente el crisantemo y la alstroemeria con un $12 \%$ y un $5 \%$, respectivamente (ICA 2018b). La Sabana de Bogotá se ha centrado en producir rosas, clavel y alstroemerias, mientras que en Antioquia se producen crisantemos y hortensias y, en el Eje Cafetero, se concentran el follaje y las flores tropicales (Ministerio de Agricultura y Desarrollo Rural 2020). No obstante, la interceptación de plagas de importancia cuarentenaria en los países importadores, son una de las principales amenazas al sector y genera cuantiosas pérdidas a los productores y comercializadores del país. En años recientes, las hortensias son el cultivo que ha presentado los mayores incrementos en áreas sembradas y exportación en el país, sin embargo también presenta los mayores índices de interceptaciones en el mercado (ICA 2020).

Para hortensias se registraron ante el ICA 1.532 predios en septiembre de 2018, en aproximadamente 1.500 ha, frente a 790 de crisantemo en el oriente antioqueño (ICA 2018b). Sin embargo, la cifra de los cultivos de hortensia podría ser mayor, dada la informalidad estimada de los predios aún sin registro (ICA 2018a). Por otra parte, no está documentada la información referente a las variedades o cultivares sembrados y, por tanto, poco se conoce acerca de la susceptibilidad de las mismas a problemas fitosanitarios particulares. Por medio de los registros del sistema de información epidemiológica y vigilancia fitosanitaria (SISFITO), el ICA busca evaluar la información referente a plagas y enfermedades de los cultivos. Para ello, se asocia con los gremios en pro de obtener información contundente y clara sobre las plagas y enfermedades. Esto permite tomar las medidas fitosanitarias pertinentes sobre los problemas identificados que, sin un adecuado control, se convierten en problemas fitosanitarios de orden económico para los productores a nivel regional (ICA 2018b). Empero, muy poco se ha documentado sobre las principales plagas y enfermedades que afectan los cultivos en campo y las áreas de mayor susceptibilidad a las mismas en el territorio.

Los ácaros, tanto el blanco Polyphagotarsonemus latus (Banks, 1904) (Acari: Tarsonemidae) como el rojo Tetranychus urticae (Koch, 1836) (Acari: Tetranychidae) y el rosado de la fresa Phytonemus pallidus (Banks, 1901) (Acari: Tarsonemidae) atacan el cultivo de hortensia y causan daños en las hojas y pérdidas en la comercialización. Son, por tanto, considerados plagas cuarentenarias para países de destino como Estados Unidos (Osorio Soto et al. 2015). Por su parte, los trips Frankliniella occidentalis (Pergande, 1895) (Thysanoptera: Thripidae) también constituyen un problema fitosanitario en los cultivos de flores, al causar daños directos en el follaje (Arévalo et al. 2003), en las flores y en los brotes, tanto por su alimentación directa como por su oviposición sobre los tejidos, ya que generan deformaciones y decoloraciones que reducen el valor del producto por el daño estético del mismo (Rhainds et al. 2007). Los áfidos Aphis spp. (Aphididae: Hemiptera), al igual que los trips, por su aparato bucal chupador también ocasionan daños en el follaje. Deforman las hojas de la flor, en los brotes donde producen enrollamiento de los tejidos y en las flores donde son encontrados al sacudir la flor por las Organizaciones Nacionales de Protección Fitosanitarias (ONPF) en los puestos de inspección (Simbaqueba et al. 2014).
Las babosas Deroceras reticulatum Müller, 1774 (Pulmonata: Stylommathophora) también han entrado a ser parte de las plagas del cultivo de hortensia, ya que se encuentran tanto en campo como en los procesos de poscosecha. Estos moluscos, aparte de ser grandes defoliadores, también pueden afectar la comercialización, ya que son indeseados en productos despachados a otros países (Osorio Soto et al. 2015). Sin embargo, aunque estos grupos taxonómicos son reconocidos de manera empírica por técnicos y productores, no han sido aún documentados en la literatura como plagas del cultivo, ni las incidencias que pueden alcanzar en los diferentes cultivares comercializados. Por este motivo, es necesario dirigir esfuerzos a estudiar los problemas fitosanitarios del cultivo, proporcionando información útil para los productores y asistentes técnicos involucrados con los cultivos de hortensia de la región.

En este trabajo se evaluaron las plagas presentes en flores de hortensia a ser comercializadas y exportadas en el oriente antiqueño, con el fin de aproximarse a la identificación de las plagas prevalentes asociadas al cultivo. Los resultados de este estudio son un aporte, no solo para los productores de la región, sino también para el ente regulador nacional y los asistentes técnicos involucrados con esta flor de exportación.

\section{Materiales y métodos}

Este estudio se realizó en la comercializadora Latin Flowers Farms S.A.S C.I, ubicada en la vereda Las Lomitas, municipio de La Ceja (Antioquia), entre abril y julio de 2018. Se inspeccionaron un total de 6.272 cajas de hortensias a ser comercializadas por la empresa, procedentes de 86 predios proveedores de los municipios de La Unión, La Ceja, Abejorral, El Retiro, Rionegro y El Carmen de Viboral y que incluyeron los cultivares más prevalentes para la comercialización como lo son Lemon, White, Azul bogotana, Blue, Purple, Shocking, Mini green, Emerald y Dark green. La unidad muestral constó de cinco flores tomadas al azar de las 50 en promedio que contiene cada una de las cajas que pasan por inspección en la poscosecha. Los datos se recopilaron en hojas de cálculo para su posterior análisis.

Inicialmente, se creó una base de datos con los campos a registrar durante el periodo de toma de muestras. Estos campos incluyeron: número de la muestra, cultivar de hortensia evaluada, número de la caja donde se tomaron las muestras, el código de la finca donde proviene la flor, la fecha de revisión y los organismos plaga encontrados: ácaros, huevos de ácaro, áfidos, trips y babosas. Los campos de esta base de datos se ajustaron de acuerdo a las tablas implementadas en campo en los cultivos de hortensia, donde las plagas de mayor prevalencia son los ácaros, áfidos, trips, babosas, chinches y cucarrones (Osorio Soto et al. 2015) estos dos últimos, dada su baja incidencia, se incluyeron como "otros" en los registros donde también se registraron otros individuos encontrados en la inspección de la flor.

Por otra parte, se hizo una colección de referencia con muestras entomológicas de los predios evaluados; los especímenes se recolectaron con agujas entomológicas y se almacenaron en tubos épendorf de 1,5 $\mathrm{ml}$ con alcohol al 70 $\%$. Cada tubo se marcó con el código de la finca, número de la muestra de flor donde se tomó y la identificación preliminar del artrópodo encontrado. Los tubos se almacenaron para la conformación de la colección y posterior curación de la misma. 
Se determinó la susceptibilidad de cada cultivar con base al porcentaje de muestras con presencia de individuos causales de interceptación y tablas de frecuencia. De igual forma, se determinaron las zonas y predios con mayor riesgo de interceptación, así como las especies de mayor presencia durante el periodo analizado.

Se evaluó el efecto de los predios y los cultivares sobre la incidencia de las diferentes plagas mencionadas, mediante un modelo linear generalizado (GLM); el cual se describe a continuación:

$$
Y_{i j k}=\mu+F_{j}+V_{k}+\varepsilon_{i j k}
$$

donde:

es la variable respuesta asociada al monitoreo, es el efecto de la j-ésima finca, es el efecto del k-ésimo cultivar y es el error estadístico. Se evaluaron diferentes modelos, con familias de distribución de probabilidad distintas, bajo el criterio de información de Akaike (AIC), calculado por la fórmula:

$$
\mathrm{AICc}=2 \mathrm{k}-2 \ln \mathrm{L}+(2 \mathrm{k}(\mathrm{k}+1)) /(\mathrm{n}-\mathrm{k}-1)
$$

donde:

$\mathrm{k}$ es el número de parámetros, $\mathrm{n}$ el número de puntos de datos y L la probabilidad del modelo dado los datos; un valor mínimo para AIC indica el mejor ajuste (Akaike 1974). Los análisis estadísticos se realizaron en el programa especializado R-project versión 3.5.1 (R Development Core Team 2013) y el programa PAST versión 3.13 (Hammer et al. 2001).

\section{Resultados}

Del total de muestras inspeccionadas, $540(8,61 \%)$ presentaron algún individuo limitante para la comercialización. El $75,19 \%$ y $54,26 \%$ asociadas a huevos y ninfas o adultos de ácaro, respectivamente. Los áfidos y trips representaron, respectivamente, un 5,93 \% y 4,07\%. Otros artrópodos participaron con un $2,10 \%$ entre los que se encuentran mosca blanca y ácaros depredadores, estos últimos, aunque benéficos y no considerados plaga, son causal de rechazo por las ONPF en los puertos de destino. La presencia de babosas representó un $1,66 \%$ del total de muestras. Se contabilizaron 13.166 huevos de ácaros (67,20\% de los individuos), 1.910 ácaros (32,01\%), 113 áfidos $(0,58 \%), 31$ trips $(0,16 \%), 12$ babosas $(0,06 \%)$ y 135 individuos diferentes a estos grupos $(0,61 \%)$. Una muestra procedente del municipio de La Unión, del cultivar Azul bogotana, presentó la mayor cantidad de individuos con 341 huevos de ácaro y 126 formas móviles de los mismos. La información referente a los individuos contabilizados en las muestras inspeccionadas se resume en la Figura 1.

De los individuos interceptados, se seleccionaron 53 muestras taxonómicamente representativas de los distintos artrópodos, para conformar la primera colección de referencia de plagas asociadas al cultivo de hortensia de la región. Los cultivares de mayor representación en la comercialización fueron White y Mini green, las demás representaron menos del $10 \%$ cada una. Por su parte, los cultivares con mayor interceptación en sala, fueron Blue, Azul bogotana y Shocking, todas con interceptaciones por encima del $14 \%$. La Tabla 1 resume información respecto a cultivares y sus plagas interceptadas expresada en porcentajes de afectación de cada taxón con respecto al total de muestras evaluadas.

En cuanto al análisis por procedencia, se recibieron muestras de 66 predios de La Unión, 15 de La Ceja, 5 del Carmen de Viboral, 2 de Abejorral y un predio del Retiro y Rionegro, respectivamente. Estos últimos se excluyeron dada su baja cantidad de muestras. La representación de muestras por municipio denota un patrón similar al del número de predios, con un mayor porcentaje para el municipio de La Unión (76,40 \%), seguido de La Ceja (14,65 \%), El Carmen de Viboral (4,48 \%) y Abejorral (3,19\%). No obstante, la afectación de dichas muestras mostró leves variaciones, siendo El Carmen de Viboral el de mayor porcentaje de muestras afectadas. Los mayores responsables de las interceptaciones para todos los municipios, fueron los huevos de ácaro y los ácaros en estados móviles (ninfas y adultos). Los áfidos se presentaron en mayor cantidad en muestras procedentes de Abejorral y los trips en el municipio de La Unión. La afectación por procedencia de acuerdo a las muestras según la presencia de cada taxón se encuentra en la Tabla 2.

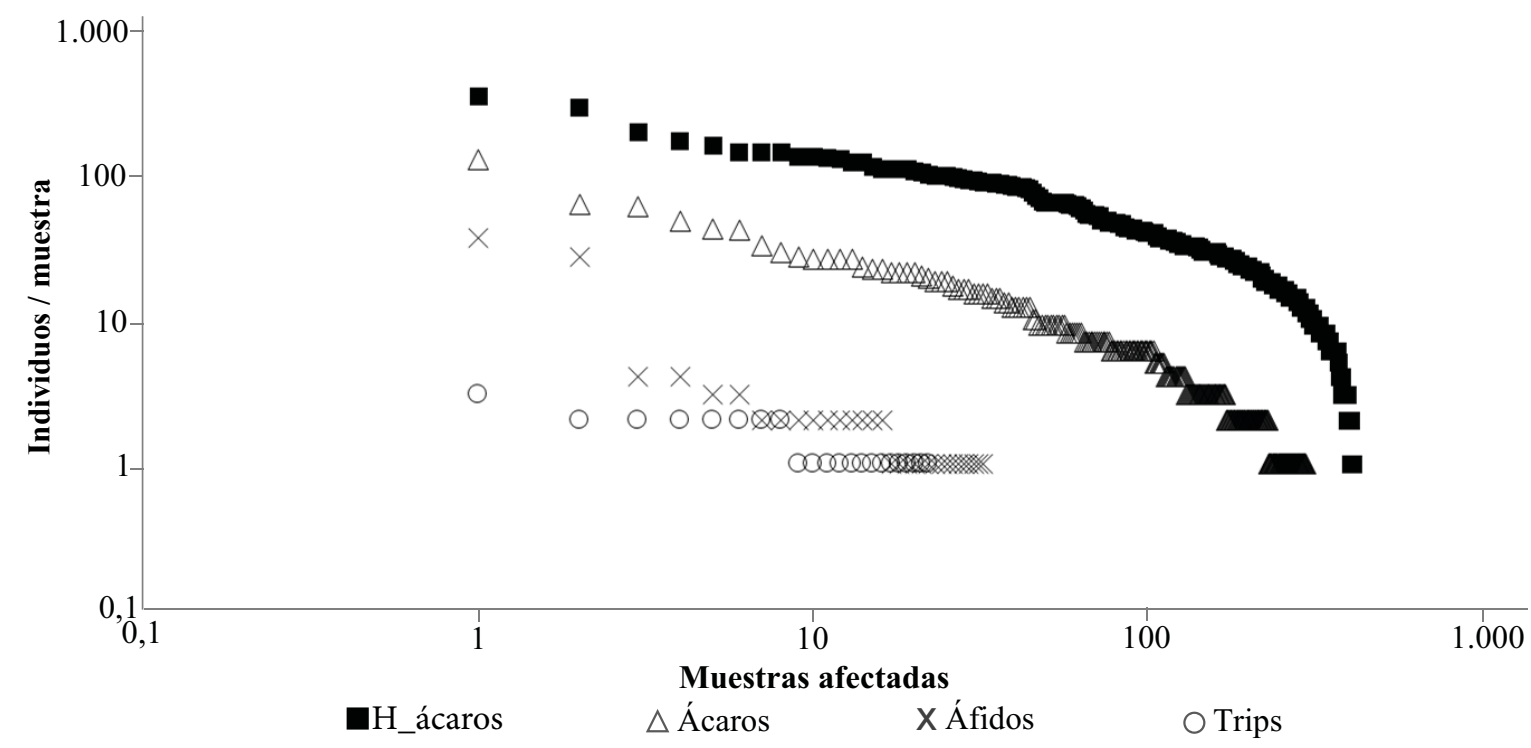

Figura 1. Abundancia de individuos plagas en muestras de flores de hortensia interceptadas para comercialización. Ejes en escala logarítmica en base 10 . 
Tabla 1. Participación porcentual en el mercado y muestras de cada cultivar de hortensia poscosecha afectadas por grupo taxonómico.

\begin{tabular}{|c|c|c|c|c|c|c|c|c|}
\hline Cultivar & $\begin{array}{c}\text { Representación en el } \\
\text { mercado }(\%)\end{array}$ & $\begin{array}{c}\% \text { de } \\
\text { Interceptación }\end{array}$ & $\begin{array}{l}\text { Huevos de } \\
\text { ácaro }\end{array}$ & Ácaros & Áfidos & Trips & Babosa & Otros \\
\hline White & 59,71 & 6,36 & 4,86 & 3,07 & 0,19 & 0,21 & 0,21 & 0,51 \\
\hline Mini green & 13,47 & $* 12,54$ & 8,99 & 7,81 & 2,01 & 0,24 & 0,12 & 0,24 \\
\hline Lemon & 7,97 & 10,80 & 8,00 & 5,80 & 0,40 & 0,40 & 0,20 & 0,80 \\
\hline Blue & 6,31 & $* 15,15$ & 12,63 & 10,10 & 0,00 & 0,25 & 0,25 & 0,76 \\
\hline Azul bogotana & 5,12 & $* 14,33$ & 10,59 & 9,03 & 0,93 & 0,62 & 0,00 & 0,62 \\
\hline Shocking & 2,93 & $* 14,13$ & 9,24 & 7,07 & 1,63 & 2,72 & 0,00 & 0,00 \\
\hline Purple & 1,82 & 3,51 & 1,75 & 0,00 & 0,00 & 0,88 & 0,88 & 0,00 \\
\hline Dark green & 1,48 & 3,23 & 2,15 & 0,00 & 0,00 & 1,08 & 0,00 & 0,00 \\
\hline Emerald & 1,18 & 4,05 & 4,05 & 1,35 & 0,00 & 0,00 & 0,00 & 0,00 \\
\hline
\end{tabular}

* Cultivares con mayores intercepciones.

El análisis por predios reveló que, durante todo el periodo de revisión, 71 presentaron huevos de ácaro, 68 ácaros móviles, 21 áfidos, 14 trips y 21 predios tuvieron presencia de otros individuos diferentes a los mencionados. Del total de predios, solo $8(9,30 \%)$ aportaron muestras libres de individuos limitantes para comercialización. Cuatro de los predios presentaron afectaciones en más del $60 \%$ de sus muestras, siete predios entre el 31 y $60 \%$ y 67 predios entre el 1 y el $30 \%$. Por otra parte, de las muestras evaluadas el $10 \%$ tuvo presencia de una sola especie limitante para comercialización, el $33,3 \%$ de dos, el $23,3 \%$ de tres, el $16,7 \%$ cuatro y el $3,3 \%$ mostraron presencia de hasta cinco taxones diferentes. Aunque más del $90 \%$ de los predios presentaron algún evento de interceptación, se destaca el hecho que no se presentó un patrón de afectación relacionado al número de muestras comercializadas por cada predio (Fig. 2).

Los datos se ajustaron a una distribución Poisson para las diferentes plagas evaluadas en el GLM, con una función de enlace logarítmica, a excepción de los huevos de ácaros cuyo mejor ajuste se dio a un modelo normal con función de enlace de identidad. En el caso de ácaros, tanto móviles como huevos, se mostraron efectos significativos tanto de predios como de los cultivares. Para los trips y los áfidos, sólo algunos cultivares presentaron efectos significativos, particularmente Shocking para ambos grupos y Mini green para áfidos. Para las babosas no se presentaron diferencias significativas en los efectos de las fincas o los cultivares. Para el conteo de ácaros móviles se encontró un efecto negativo, y altamente significativo, de los cultivares Emerald, Lemon, Shocking y White (Tabla 3), lo que indica que, en una muestra evaluada de forma aleatoria, estos cultivares tienden a presentar menor abundancia de la plaga. Para el conteo de los huevos de acaro también se encontró un efecto negativo en casi todas los cultivares a excepción de Blue y Shocking cuyas estimaciones fueron positivas, pero no significativas. Sin embargo, los cultivares Purple y White presentaron efectos negativos significativos. Con respecto al conteo de trips, solo se encontró significancia para el cultivar Shocking, con un valor positivo, sin embargo, es de notar la poca cantidad de individuos en las muestras interceptadas (Fig. 1). Para la presencia de babosas no se obtuvieron estimaciones significativas. Los efectos estimados para los cultivares en las plagas más representativas en el estudio se presentan en la Tabla 3.

\section{Discusión}

Las hortensias han colonizado un lugar privilegiado en las exportaciones de flor de corte en el Colombia (Tenjo et al. 2006) pero también, han sido el producto con más interceptaciones debido a plagas durante los últimos tres años, por parte del ICA y del Servicio de Inspección de la Salud Animal y de las Plantas de los Estados Unidos (APHIS) (ICA 2020). Sin embargo, no existe literatura publicada referente a la incidencia de plagas ni a la asociación de estas a los cultivares comercializados. Este trabajo presenta por primera vez información referente a las plagas de mayor incidencia en la comercialización de flores de hortensia tipo exportación.

Los resultados sugieren que la interceptación asociada a plagas cuarentenarias, parece estar ligada más al manejo particular en los distintos predios productores que a los cultivares o localización geográfica de los mismos. Esto es soportado por el hecho que la afectación de los distintos cultivares no se relacionó con el volumen comercializado y que municipios con menor participación mostraron mayores porcentajes de flor interceptada. No obstante, se resalta que los cultivares de flores azules (Blue, Azul bogotana y Shocking) o procedentes de materiales azules como Mini green, presentaron mayores porcentajes de afectación que cultivares de otros colores (Tabla 1).

Tabla 2. Porcentaje de afectación por organismo plaga en flores de hortensia poscosecha, para los diferentes municipios evaluados.

\begin{tabular}{ccccccccc}
\hline Municipio & $\begin{array}{c}\text { Total } \\
\text { muestras (n) }\end{array}$ & $\begin{array}{c}\text { muestras } \\
\text { afectadas }\end{array}$ & Ácaros & $\begin{array}{c}\text { Huevos de } \\
\text { ácaro }\end{array}$ & Áfidos & Trips & Babosa & Otro \\
\hline Abejorral & 200 & 11,00 & 31,82 & 50,00 & 4,55 & 0,00 & 0,00 & 4,55 \\
El Carmen de Viboral & 281 & $* 17,08$ & 39,58 & 54,17 & 2,08 & 0,00 & 0,00 & 2,08 \\
La Ceja & 919 & 9,68 & 31,46 & 50,56 & 1,12 & 1,12 & 2,25 & 6,74 \\
La Unión & 4792 & $* 15,80$ & 30,91 & 41,74 & 3,83 & 2,64 & 1,32 & 2,91 \\
\hline
\end{tabular}




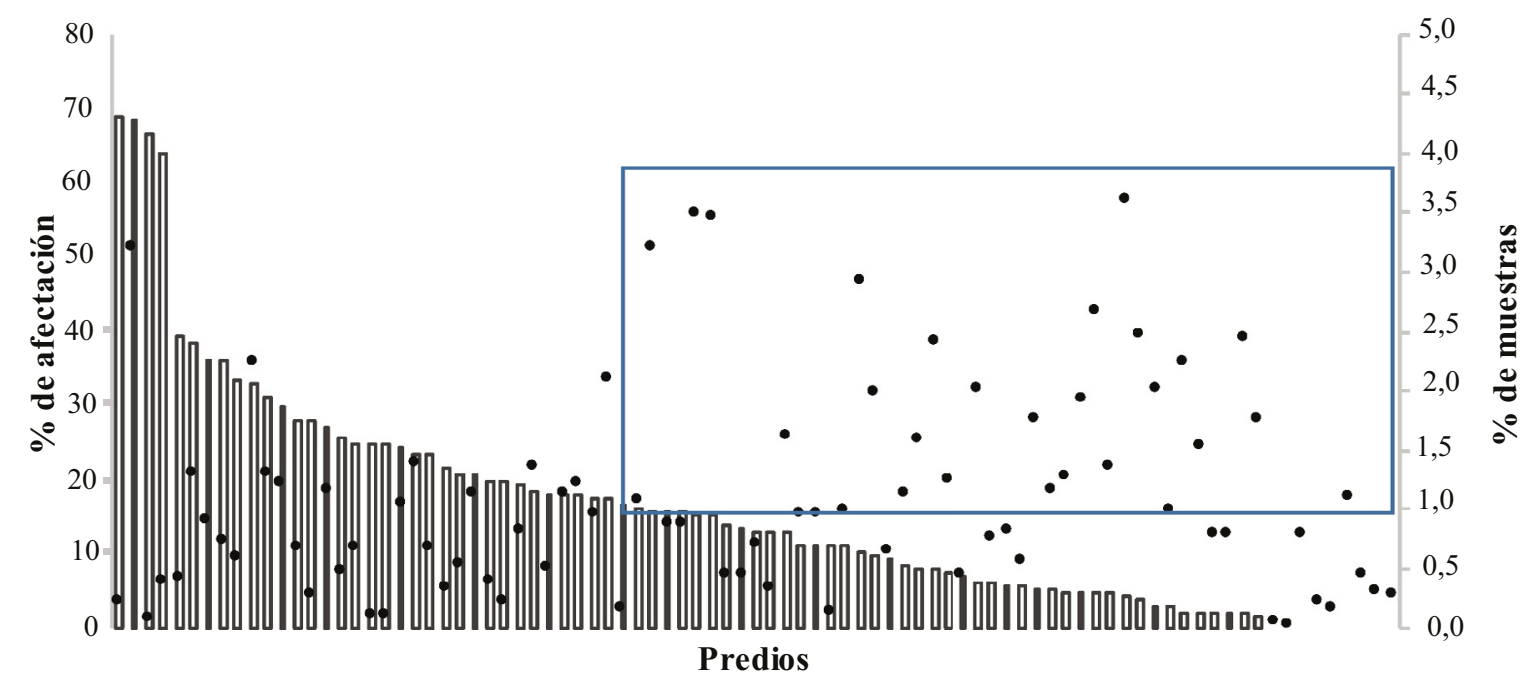

Figura 2. Porcentaje de muestras afectadas por predio (barras, eje izquierdo). Porcentaje de muestras totales por predio evaluadas en el estudio (puntos, eje derecho).

Los ácaros, tanto en huevos como estados móviles, fueron los individuos de mayor interceptación en los distintos cultivares como en los municipios, lo que soporta la creencia no documentada de técnicos y productores sobre la afectación de sus cultivos en el campo. Sin embargo, para este grupo de plagas el número de individuos promedio por muestra interceptada mostró cultivares con promedios elevados en flores de otra coloración como Lemon y White (Fig. 3), lo que sugiere que no hay asociación particular a los distintos cultivares.

Al realizar comparaciones de muestras de varios cultivares procedentes de los mismos predios, en busca de reducir la variación espacial, no se encontraron patrones de afectación por cultivar, sin embargo, la mayoría de muestras de White presentaron afectaciones. En este sentido, algunas excepciones son de resaltar. Por ejemplo, uno de los predios evaluados aportó 70 muestras $(1,11 \%$ del total) de siete cultivares distintos, sin interceptaciones por plagas, lo que demuestra que un buen manejo al interior de los cultivos y los tratamientos poscosecha, pueden disminuir los niveles de afectación en la comercialización sin estar ligados a los cultivares vendidos.
Por ejemplo, para los ácaros tanto en huevos como en sus formas móviles, una de las medidas implementadas en poscosecha para su eliminación, es la inmersión en ovicidas, insecticidas $\mathrm{y} / \mathrm{o}$ acaricidas. Esta práctica puede tener mejor efecto en formas móviles de los ácaros y, aunque tienen también un efecto negativo sobre los huevos, la presencia de éstos, sean vivos o muertos, es causal de rechazo en comercialización y esto puede explicar las mayores abundancias en las muestras interceptadas (Fig. 1). Otro tratamiento en la poscosecha, es agitar el material a comercializar, no obstante, esta medida no es igualmente efectiva para todos los artrópodos. Por ejemplo, la morfología de las patas de los ácaros (Kakar et al. 2017), les permite aferrarse a los tejidos de la planta más firmemente que áfidos y trips, los cuales caen fácilmente con estos movimientos. De igual manera, se esperaría que esta práctica, no tenga efectos sobre los huevos de los ácaros depositados sobre las hojas. Así pues, las grandes diferencias en la incidencia de las distintas plagas en la comercialización, pueden ser resultado del manejo poscosecha realizado por los productores.

Tabla 3. Estimativas y errores estándar para el efecto de los cultivares en la atracción de diferentes plagas de hortensia en poscosecha.

\begin{tabular}{ccccccccc}
\hline \multirow{2}{*}{ Cultivar } & \multicolumn{2}{c}{ Ácaros } & \multicolumn{2}{c}{ Huevos de ácaro } & \multicolumn{2}{c}{ Trips } & \multicolumn{2}{c}{ Babosas } \\
\cline { 2 - 8 } & Estimado & E. E & Estimado & E. E & Estimado & E. E & Estimado & E. E \\
\hline Azul bogotana & $-3,368^{* * *}$ & 1,001 & $-1,2704$ & 1,8085 & $-0,2295$ & 0,0096 & $-0,4263$ & 0,0001 \\
Blue & 0,019 & 0,106 & 0,4236 & 0,9645 & $-0,898$ & 1,166 & 19,040 & 5022 \\
Dark green & $-17,050$ & 341,700 & $-3,3418 *$ & 1,5093 & 0,340 & 1,342 & 1,834 & 10670 \\
Emerald & $-3,585^{* * *}$ & 1,004 & $-3,2583 *$ & 1,6313 & $-18,200$ & 7202,000 & 0,139 & 12390 \\
Lemon & $-0,6278^{* * *}$ & 0,118 & $-1,0051$ & 0,9368 & $-0,470$ & 0,971 & 17,870 & 5022 \\
Mini green & $-0,139$ & 0,088 & $-0,3156$ & 0,8463 & $-1,160$ & 1,049 & 16,510 & 5022 \\
Purple & $-18,430$ & 411,700 & $-8,9749 * * *$ & 1,7434 & 0,996 & 1,925 & 19,360 & 5022 \\
Shocking & $-0,5406 * *$ & 0,188 & 0,8730 & 1,3293 & $2,058 *$ & 0,959 & 0,340 & 8739 \\
White & $-1,296 * * *$ & 0,088 & $-2,3476 * *$ & 0,7659 & $-0,538$ & 0,708 & 19,020 & 5022 \\
\hline
\end{tabular}

E. $\mathrm{E}=$ Error estándar. Los mayores efectos se resaltan con asteriscos: * Efecto significativo $(\mathrm{P}<0,05)$. ** Efecto muy significativo $(\mathrm{P}<0,01)$. *** Efecto altamente significativo $(\mathrm{P}<0,001)$. 


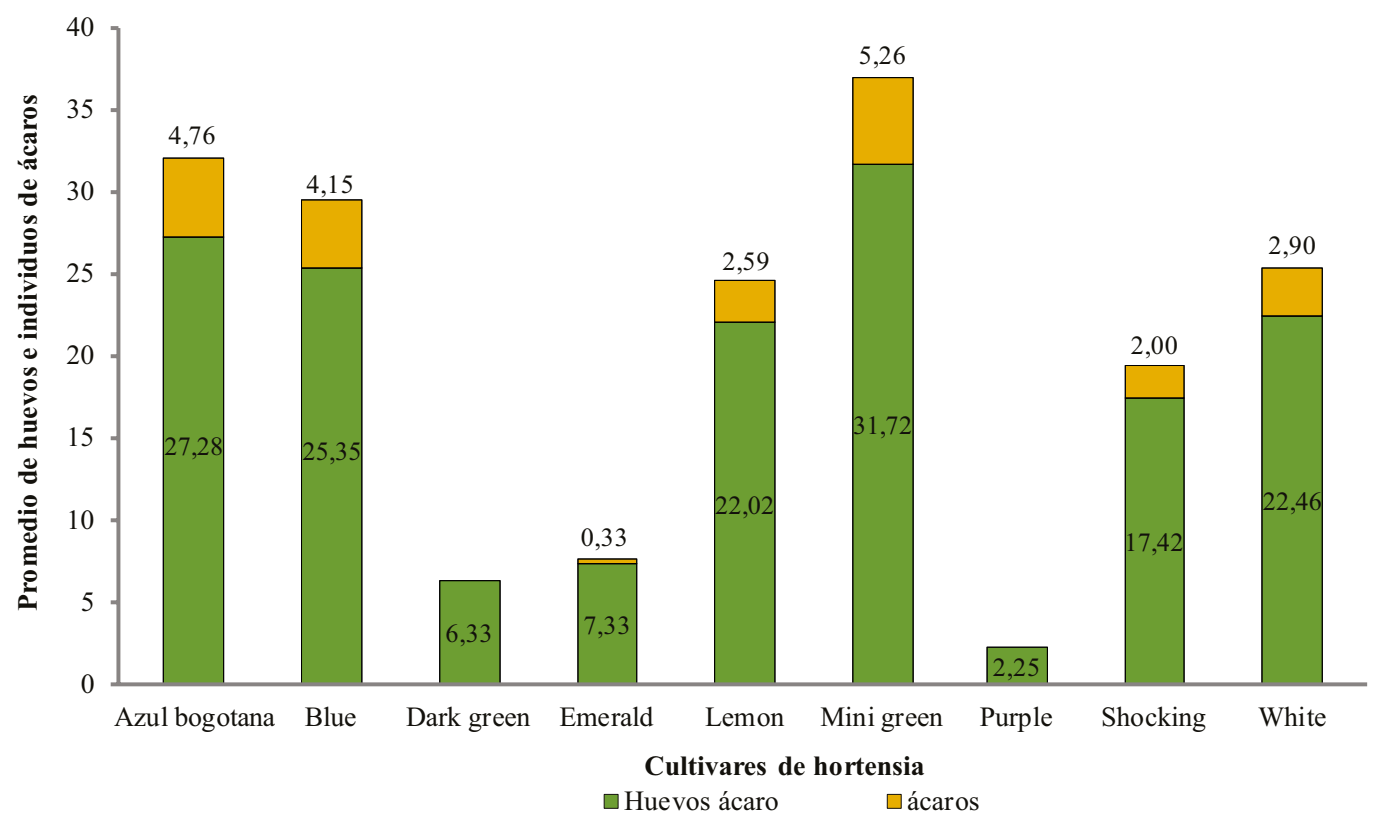

Figura 3. Promedio de presencia de ácaros en estados móviles y huevos de ácaros en muestras afectadas por éstos en los nueve cultivares evaluados.

Respecto a los municipios de procedencia de la flor, El Carmen de Viboral fue el de mayor porcentaje de afectación con respecto a la cantidad de muestras revisadas, a pesar de no ser el de mayor número de muestras aportadas, ni el de más predios registrados en la comercializadora. No obstante, según los datos del ICA, para septiembre del 2018, es el municipio con mayor cantidad de predios productores de hortensia registrados ante dicha entidad. Los predios que tuvieron la mayor cantidad de muestras evaluadas ( $>1 \%$ de muestras totales), presentaron en su mayoría porcentajes de afectación inferiores al $15 \%$, con algunas excepciones notables (Fig. 1). Indirectamente, la cantidad de muestras podría ser un indicador del tamaño de los cultivos y a su vez de su nivel de tecnificación, tanto en campo como en poscosecha, con lo cual se esperarían menores afectaciones de la flor al momento de su inspección. Sin embargo, esta idea debe ser soportada por evaluaciones directas en campo en el futuro. Aunque los datos aquí recopilados representan información procedente de solo el 5,6\% de los predios de Antioquia registrados ante el ICA, es un primer paso para dirigir esfuerzos en la evaluación de la incidencia de plagas de hortensia en el campo.

Aunque aún no se cuenta con buena información sobre la susceptibilidad a plagas de los distintos cultivares en el campo, la presencia de individuos en el material de exportación puede ser información cuya interpolación permita identificar plagas, sectores y cultivares prioritarios para inspección y evaluación en campo. Es de mencionar también, que otros artrópodos encontrados en el monitoreo incluyen especies que no son consideradas plaga, como los ácaros depredadores, que a pesar de ser benéficos en los cultivos, son también causal de interceptación y rechazo de la flor por las ONPF en los puertos de destino al presentarse en el momento de la inspección (Food and Agriculture Organization of the United Nations 2002). Esto tiene implicaciones que afectarían los programas de manejo integrado de plagas que se realizan en los cultivos actualmente. Los datos aquí publicados, son de gran importancia para productores, asistentes técnicos involucrados con el cultivo y personal del ente gubernamental regulatorio que, a la fecha, le han dado un manejo al cultivo sin investigación técnica detallada que soporte el manejo del mismo, y que han traído como consecuencia, los altos índices de interceptación en los puertos de destino.

\section{Conclusiones}

La incidencia de plagas en las flores de hortensias evaluadas, destinadas a exportación, no responde a los cultivares o procedencia geográfica de los mismos (municipios). Por el contrario, se sugiere que la presencia de organismos sujetos a interceptación, se relaciona al manejo particular en los distintos predios productores y al manejo poscosecha previo a la comercialización realizado en los mismos. Por tanto, la revisión del material de hortensias en el proceso de comercialización, es un proceso fundamental antes de la exportación, previniendo así las interceptaciones y sanciones posteriores al país.

\section{Agradecimientos}

A German Danilo Posada Villada y la empresa Latin Flowers Farms S.A.S C.I, quienes permitieron la realización de este trabajo. Este trabajo fue apoyado por el Sistema de Investigación y Desarrollo, convocatoria 2016 de la Universidad Católica de Oriente, Antioquia-Colombia.

\section{Literatura citada}

AKAIKE, H. 1974. A new look at the statistical model identification. IEEE Transactions on Automatic Control 19 (6): 716-723. https://doi.org/10.1109/TAC.1974.1100705

ARBELÁEZ, G. 1993. La floricultura colombiana de exportación. Agronomía Colombiana 10 (1): 5-11. https://revistas.unal.edu. co/index.php/agrocol/article/view/21224/22205

ARÉVALO P., E.; QUINTERO F., O. X.; CORREA L., G. 2003. Reconocimiento de trips (Insecta: Thysanoptera) en floricultivos de tres corregimientos del municipio de Medellín, Antioquia (Colombia). Revista Colombiana de Entomología 29 (2): 169-175. http://www.scielo.org.co/pdf/rcen/v29n2/v29n2a09.pdf

FOOD AND AGRICULTURE ORGANIZATION OF THE UNITED NATIONS (FAO). 2002. Aplicación de medidas integradas 
en un enfoque de sistemas para el manejo del riesgo de plagas. Food and Agriculture Organization of the United Nations. http:// www.fao.org/3/a-y4221s.pdf

HAMMER, Ø.; HARPER, D. A. T.; RYAN, P. D. 2001. PAST: Paleontological statistics software package for education and data analysis, $178 \mathrm{~kb}$. Palaeontologia Electronica 4 (1): 1-9. https://palaeo-electronica.org/2001_1/past/issue1_01.htm https://palaeo-electronica.org/2001_1/past/past.pdf

INSTITUTO COLOMBIANO AGROPECUARIO (ICA). 2018a. El ICA apoya el florecimiento del sector ornamental de Antioquia. Instituto Colombiano Agropecuario, marzo. Disponible en: https:// www.ica.gov.co/noticias/ica-apoya-exportacion-ornamentalantioquia [Fecha revisión: julio 2018]

INSTITUTO COLOMBIANO AGROPECUARIO (ICA). $2018 \mathrm{~b}$. Lista de empresas exportadoras y predios productores de ornamentales de exportación. SISFITO, Antioquia. Instituto Colombiano Agropecuario. Disponible en: https://sisfito.ica.gov.co/ reportes/reporte_inicio_I.php [Fecha revisión: mayo 2018]

INSTITUTO COLOMBIĀNO AGROPECUARIO (ICA). 2020. Polillas y otros artrópodos de atención prioritaria para los productores de hortensia (Hydrangea spp.). Alerta temprana fitosanitaria Boletín No 18, Subgerencia de Protección Vegetal del ICA, Agosto 2020. https://www.ica.gov.co/getattachment/ ICAComunica/alertasfito/ALERTA_TEMPRANA_HORTENSIA_06082020.pdf.aspx?lang=es-CO [Fecha revisión: agosto 2020]

KAKAR, H.; IQBAL, A.; KAMRAN, K.; ARIF, S.; SHAHEEN, U.; KAMRAN TAJ, M.; SAMAD, A.; SHAHABUDDIN.; AHMED ESSOTE, S.; ALAM, R.; ALI, A. 2017. Morphometric study of Tetranychus urticae (Acariformes: Tetranychidae) in Ziarat district, Baluchistan, Pakistan. International Journal of Biosciences 10 (1): 401-407. https://dx.doi.org/10.12692/ijb/10.1.401-407

MINISTERIO DE AGRICULTURA Y DESARROLLO RURAL (MINAGRICULTURA). 2020. Cadena de flores. Dirección de Cadenas Agrícolas y Forestales, Ministerio de Agricultura y Desarrollo Rural, Febrero 2020. https://sioc.minagricultura.gov.co/ Flores/Documentos/2019-12-30\%20Cifras\%20Sectoriales.pdf [Fecha revisión: agosto 2020]

OSORIO SOTO, G.; VILLA CASTRO, J.; ARREDONDO URREGO, J.; VÁSQUEZ MARTÍNEZ, J.; RESTREPO OSPINA, J.; RESTREPO MEJÍA, L.; JARILLO GIL, W. 2015. Manual técni- co del cultivo de hortensias de exportación bajo buenas prácticas agrícolas. Medellín, Colombia, $130 \mathrm{p}$.

RHAINDS, M.; DOYON, J.; RIVOAL, J.; BRODEUR, J. 2007. Thrips-induced damage of chrysanthemum inflorescences: evidence for enhanced leakage of carotenoid pigments. Entomologia Experimentalis et Applicata 123 (3): 247-252. https://doi. org/10.1111/j.1570-7458.2007.00543.x

SIMBAQUEBA, R.; SERNA, F.; POSADA-FLÓREZ, F. J. 2014. Curaduría, morfología e identificación de áfidos (Hemiptera: Aphididae) del Museo Entomológico Unab. Primera Aproximación. Boletín Científico Centro de Museos de Historia Natural 18 (1): 222-246. http://www.scielo.org.co/pdf/bccm/v18n1/ v18n1a17.pdf

TENJO G., F.; MONTES U., E.; MARTÍNEZ T., J. 2006. Comportamiento reciente (2000-2005) del sector floricultor colombiano. Colombia. 22 p. http://www.banrep.gov.co/docum/ftp/borra363. pdf

VALCÁRCEL-CALDERÓN, F. 2013. El control biológico de plagas en la floricultura colombiana. Simposio 6 "Plagas de las flores”. En: Rueda-Ramírez, D.; Torrado-León, E.; Becerra, E. H. (Comp.). Libro de memorias. 40 Congreso Socolen. Bogotá, D.C., 10, 11 y 12 de julio de 2013. Sociedad Colombiana de Entomología. CD-ROM. Bogotá, D. C., 445 p. http://www.socolen.org.co/images/stories/pdf/40_Congreso.pdf

\section{Origen y financiación}

El presente artículo derivó del trabajo de grado de la estudiante de Agronomía María Daniela Molina Acosta, de la Facultad de Ciencias Agropecuarias de la Universidad Católica de Oriente, adscrito al proyecto de investigación 201634 "Reconocimiento de plagas y enfermedades asociadas a cultivos de Hydrangeas sp." de la convocatoria interna de Investigación y Desarrollo UCO -2016.

\section{Contribución de los autores}

María Daniela Molina Acosta y Carlos Eduardo Giraldo Sánchez diseñaron del estudio y redactaron el manuscrito. María Daniela Molina Acosta recolectó el material biológico. Todos los autores analizaron los datos y participaron en la revisión y edición del manuscrito. 